\title{
NUTRICIÓN HUMANA, MEJORAMIENTO VEGETAL Y METABOLISMO SECUNDARIO: UNA REVISIÓN
}

\author{
HUMAN NUTRITION, PLANT BREEDING AND \\ SECONDARY METABOLISM: A REVIEW
}

Marco Cabezas Gutiérrez ${ }^{1}$

RESUMEN

Los alimentos de origen vegetal contienen una amplia variedad de vitaminas, minerales y metabolitos únicos, que pueden tener efectos beneficiosos o detrimentales en la salud y el bienestar de los humanos. Una mezcla de carbohidratos, lípidos, aminoácidos, proteínas, 17 elementos minerales y 13 vitaminas son componentes esenciales de la dieta. En países en desarrollo, el problema de hambre y de desnutrición es cada día más preocupante; aspecto distinto es el encontrado en los países avanzados donde la problemática de salud se debe principalmente a enfermedades coronarias y afecciones debidas al sobrepeso. La ingeniería genética, la proteómica, la ingeniería metabólica y otras herramientas del fitomejoramiento, han sido enfocadas para aumentar la calidad y la inocuidad de los alimentos de origen vegetal. En el presente artículo, se hace una revisión de los principales grupos de compuestos considerados esenciales en la salud y en la alimentación humana, la forma en que pueden ser mejorados y las perspectivas de su utilización hacia un futuro mediato.

Palabras clave: Vitaminas, folatos, pantotenato, fitomejoramiento, resveratrol.

${ }^{1}$ Ingeniero Agrónomo. M.Sc. Universidad de Ciencias Aplicadas y Ambientales U.D.C.A. Dirección para correspondencia: mcabezas@udca.edu.co
SUMMARY

Plant-derived foods contain a wide variety of vitamins, minerals and unique metabolites, which may have beneficial or detrimental effects on human health and well-being. A mixture of carbohydrates, lipids, amino acids, proteins, 17 mineral elements and 13 vitamins are the essential components of the diet. In developing countries the problem of hunger and malnutrition is of mayor concern every day, being a different aspect the one found in developed countries where the health problem is due mainly to coronary diseases and affections due to overweight. Genetic engineering, the proteomic, the metabolic engineering and other tools of plant breeding, have been focused to increase the quality and the innocuity of food of plant origin. In this article the main groups considered essential compounds for the health and the human feeding, the form in which they can be improved and perspectives of their use within a mediate future is revised.

Key words: Vitamins, folates, pantothenate, plant breeding, resveratrol.

\section{INTRODUCCIÓN}

Las primeras tres décadas del siglo XX fueron llamadas la edad de oro de las ciencias nutricionales, debido a que la gran mayoría de los requerimientos en la dieta humana, para mantener buena salud y óptimo estado nutricional, fueron descubiertos. Las vitaminas, los minerales, los aminoácidos y los ácidos grasos necesarios para aplacar los síntomas de hambre y de desnutrición fueron identificados y caracterizados y se estimaron las 
cantidades de nutrimentos requeridas para satisfacer dichas necesidades. Sin embargo, en la actualidad, la deficiencia de micronutrientes y de vitaminas continúa limitando la adecuada nutrición de la población, especialmente en los países pobres. Caso contrario, se nota en los países avanzados, allí los esfuerzos gubernamentales, las condiciones sociopolíticas y las culturales han erradicado el hambre en alta proporción y se ha conseguido disminuir la mortalidad, por efectos nutricionales. El problema en el mundo desarrollado parece ser la alta incidencia de enfermedades coronarias, el cáncer en sus distintas manifestaciones, la diabetes y la obesidad (Oltmans et al. 2005; Xiao et al. 2006).

Los estudios demostraron cómo el consumo de frutas y de verduras ha disminuido los riesgos y las enfermedades en el mundo occidental y se correlacionan negativamente con los niveles de cáncer y enfermedades cardiovasculares. No obstante, muy pocos son los lugares en el planeta donde el consumo de estos alimentos es el adecuado. Estas causas hacen pensar que, ya despejado el camino para encontrar la cura a los problemas nutricionales del mundo, se deba entonces utilizar las herramientas aportadas por los avances de la ciencia y la tecnología, en términos de producción de alimentos suficientes y de alta calidad. La agricultura y más aún una de sus disciplinas, el mejoramiento genético de plantas cultivadas es una de esas herramientas. Se conocen varios esfuerzos por obtener alimentos enriquecidos y fortificados, producto de la manipulación genética de las plantas, más que por el uso de tecnologías agroindustriales. El arroz conocido como "Golden Rice", los maíces modificados genéticamente para incrementar los niveles de proteína, las nuevas variedades de canola, papa y tomate, con mayores contenidos de carotenoides, son algunos de los ejemplos (Ghandilyian et al. 2006; Schaub et al. 2005).

En el presente documento, se presentan algunos de los principales aspectos que, en la actualidad, se manejan para mejorar la calidad alimenticia de las especies agrícolas, la relación de estos procesos con el metabolismo vegetal y las tendencias actuales de aspectos, como la biofortificación, el enriquecimiento con micronutrientes, el incremento de antioxidantes y la actividad de las vitaminas y cofactores bioquímicos.

Especial atención, se presta a algunos alimentos y bebidas de origen tropical, como el café y el chocolate, así como también a los efectos benéficos de los vinos y las hortalizas crucíferas buscando así contextualizar al lector en la relación metabolismo secundario, calidad nutricional y mejoramiento genético de plantas cultivadas.

\section{Fortificación de alimentos}

Ha sido considerada como la mejor estrategia a largo plazo para prevenir las deficiencias nutricionales, especialmente en áreas desarrolladas (Johns E Eyzaguirre, 2007). Consiste en adicionar en el proceso postcosecha y/o agroindustrial, los elementos nutricionales requeridos para balancear las dietas de la población (White $\mathcal{E}$ Broadley, 2005). En los países en desarrollo puede presentar algunas dificultades, especialmente debido a la falta de infraestructura, como laboratorios especializados en biofortificación y a la discontinuidad de los programas, tal y como sucedió con el mejoramiento del maíz en Colombia (Datta et al. 2003; Lucca et al. 2006).

El hierro es el mineral de más difícil suplementación, debido a algunos problemas técnicos derivados de la solubilidad de compuestos ferrosos. En ocasiones, la reacción del hierro ferroso produce colores indeseables en los productos, afectando la presentación de los mismos (López \& Martos, 2004).

\section{Biofortificación}

Comparado con los alimentos fortificados, las plantas biofortificadas tienen grandes ventajas, debido a que no necesitan procesamientos específicos una vez almacenados y no requieren infraestructura especial (Welch $\varepsilon$ Graham, 2004; White E Broadley, 2005). Sin embargo, las variedades de cereales con altos micronutrientes no son valoradas como debiera ser en la alimentación humana. Al incrementar la eficiencia en la toma de nutrientes del suelo, se puede también alcanzar una mayor resistencia a enfermedades (Bouis, 2003). La biofortificación, se puede desarrollar a partir de las siguientes estrategias (King, 2002):

Mejoramiento tradicional. Según Lucca et al. (2006), el IRRI en poblaciones segregantes de arroz, encontró variaciones en la concentración de Fe entre 7,5 y $24,4 \mathrm{mg} \cdot \mathrm{kg}^{-}{ }^{1}$. Esto refleja, la importancia de identificar genotipos de buena concentración del mineral y buena disposición del material genético para incrementar los niveles del nutrimento. Las reducciones de ácido fítico, uno de los principales antinutrientes, fueron parte de la causa de los aumentos de hierro, pero se debe tener 
en cuenta que el ácido fítico es fundamental como despensa de fósforo en la germinación de las semillas, hecho contraproducente desde el punto de vista agrícola (Duhan et al. 2002).

Modificaciones genéticas. La concentración de micronutrientes en los tejidos de plantas agrícolamente aprovechables están altamente controlados por mecanismos homeostáticos, los cuales son responsables de la absorción, la traslocación y la redistribución de minerales en los diferentes tejidos (Welch E Gram, 2004). La primera barrera, se encuentra en la interfase suelo-raíz. Con base en este aspecto, las plantas han desarrollado dos tipos de estrategias: las dicotiledóneas y las monocotiledóneas no gramíneas, sobreregulan la reducción del hierro, el hierro ferroso transportado y las ATPasas dependientes de protones (Johns \& Eyzaguirre, 2007). Las plantas gramíneas segregan unos compuestos de bajo peso molecular llamados fitosideróforos y una vez tomado el hierro por estos accesorios es llevado vía xilema, transportado al floema y de allí a los órganos de almacenamiento para ser aprovechado en la alimentación humana (Meng \& Yang, 2005).

\section{Biofortificación del arroz como comida básica}

El arroz es el alimento básico para los habitantes en más de 33 países, cuyos pobladores son dependientes directos de este grano. Se considera el cereal más importante del mundo, pues provee el $80 \%$ de las calorías para unos 3.000 mil millones de personas, la mitad de la población mundial. En arroz, como en todos los cereales, la gran mayoría de micronutrientes se encuentran almacenados en la cubierta, la aleurona y el embrión. El gran inconveniente radica en que en los procesos de molinería estas partes del grano son eliminadas por tener altos componentes lipídicos que, una vez descompuestos, producen ranceamiento y, por tanto, daños en la calidad del producto almacenado. Con el trillado, se pierde gran proporción de nutrimentos. El endospermo tiene bajas cantidades de vitamina A y muy limitados contenidos de hierro y otros nutrimentos (Schaub et al. 2005; Lucca et al. 2006).

Los avances en la ingeniería genética, se han concentrado en darle calidad al grano mediante la introducción de hierro y de enzimas capaces de sintetizar $\beta$-caroteno en el endospermo. Producto de ello es el grano denominado "Golden Rice" (GR), el cual fue obtenido mediante transformación genética de una variedad de Oriza sativa L. tipo japónica. El grano de GR es de color amarillo, gracias a que en el endospermo se pudo introducir un gen capaz de sintetizar la provitamina A, haciendo del grano un producto prometedor para disminuir las deficiencias de la vitamina A, especialmente, en países en desarrollo (Meng \& Yang, 2005).

La introducción de la vía del $\beta$-caroteno en el endospemo de los granos de arroz, se logró a través de la activación de la fitoenosintasa, para producir el producto intermedio fitoeno proveniente de geranil-geranil-difosfato (GGDP). La síntesis de $\beta$-caroteno requiere de otros tres pasos a saber: acción de la fitoeno desaturasa, acción de la $\zeta$-saturasa y acción del licopeno- $\beta$ ciclasa. Las saturasas son activadas por una enzima presente en la bacteria Erwinia carotovora, la cual fue usada mediante técnicas de ingeniería genética para proveer las enzimas en el endospermo del arroz y así darle la coloración amarilla, producto de la presencia del pigmento antes referenciado (Al Babili \& Beyer, 2005).

\section{Biosíntesis de la vitamina $B_{2}$.}

Con base en lo expuesto por Fischer $\mathcal{E}$ Bacher (2006) y Schramek et al. (2001), la riboflavina, Vitamina $B_{2}$ es el precursor universal de la flavocoenzima rivoflavin monofosfato (FMN) y de flavin-adenin dicnucleótido (FAD), importantes cofactores en la cadena de transferencia de electrones, en la actividad membranar y en la producción de energía en varios organelos. Los microorganismos y los vegetales las sintetiza, pero los animales y el hombre deben tomarla de la dieta.

La biosíntesis de la riboflavina requiere una molécula de guanocin tri fosfato (GTP) y dos moléculas de ribulosa 5-fosfato (Bacher et al. 2000). El anillo imidazol del GTP es abierto de forma hidrolítica, produciendo 2,5-diaminopirimidina, la cual es convertida a 5-amino-6-ribitilamino-3,4(1H,3H)-pirmidinediona. La condensación de 3,4-dihidroxi-2-butanona 4-fosfato, obtenida de la ribulosa 5-fosfato, produce 6,7-dimeti-8-ribitidillumacina. La desmutación de de este compuesto da como resultado 5-amino-6-ribitidil amino $(1 \mathrm{H}, 3 \mathrm{H})$-pirimidiona, la cual se recicla para recuperar la vía. La vía biosíntética en plantas y en eubacterias es muy similar, no sucede lo mismo con lo reportado para hongos y arquea (Fischer E Bacher, 2005). 
Se han encontrado once enzimas comprometidas con la síntesis de vitamina $\mathrm{B}_{2}$ en plantas: GTP ciclohidrolasa, 5-fosfato de aminasa, 5-fosfatoreductasa en dos pasos distintos, pirimidin dienona-5-fosfatodeaminasa, una fosfatasa, 2-butanona-4 fosfato sintasa, dietil 8-ribitil lumazinasintasa, rivoflavinsintasa, rivoflavinkinasa y FAD sintasa (Fischer E Bacher, 2006).

\section{Biosíntesis del pantotenato en plantas superiores}

El pantotenato, vitamina $B_{5}$ es un compuesto soluble en agua, el cual es precursor de $4^{\prime}$-fosfopantoteteina, coenzima A, proteínas tipo transportador, todos cofactores esenciales en muchos procesos metabólicos, claves en el organismo humano. Ejemplo de ello es el papel de la coenzima A en el ciclo de Krebs, la oxidación, la producción de lípidos y de ácidos grasos y, en el caso de plantas, la base para el metabolismo secundario, incluyendo lignina (Chakauya et al. 2006).

Se estima que alrededor de 100 enzimas requieren de pantotenato como cofactor. Los animales deben obtener esta vitamina a partir de su dieta, mientras que plantas y microorganismos la sintetizan permanentemente. Pantotenato, se deriva del griego pantos, el cual significa universal, entendiéndose así su localización en todos los tejidos de los organismos vivos. La ausencia de este metabolito en animales hace que las enzimas estén sujetas al ataque de organismos productores de tóxicos, antibióticos e incluso al ataque de moléculas de plaguicidas (Webb et al. 2004). En la industria, al año se producen alrededor de 4000 t de pantotenato, el cual es utilizado en la producción de complementos alimenticios, productos de belleza y protectores de piel, principalmente (Chakauya et al. 2006).

Los acercamientos para entender la síntesis del pantotenato en plantas son de reciente producción, de hecho, inicialmente se trabajó con base en lo encontrado para Escherichia coli. Muy posiblemente, se debió a que en vegetales existía la dificultad para identificar mutantes con bloqueo de los diferentes pasos. Las vías tanto en bacterias como en plantas tienen algo de similitud, pero varían en los tipos de enzimas involucrados y en organización de la vía (Webb et al. 2004).

La biosíntesis del pantotenato tiene dos ramas: en la primera, el $\alpha$-cetoisovalerato, se convierte en cetopan- toato por acción de la enzima cetopantoato hidroximetiltransferasa (KPHMT) y con 5,10-metilen tetrahidrofolato, como cofactor. Posteriormente, el cetopantoteato es reducido a pantoato mediante la enzima cetopantoteatoreductasa y NADPH, como cofactor. De la otra rama, una molécula de ${ }_{L}$-aspartato es transformada a $\beta$-alanina gracias a la ${ }_{L}$-aspartato- $\alpha$-decarboxilasa. $\beta$-alanina y pantoato se unen para formar pantotenato, acción catalizada por la pantotenatosintetasa, dependiente de ATP (Chakauya et al. 2006).

\section{Manipulación de los niveles de pantotenato en plantas}

La producción actual de pantotenato es principalmente de origen químico, pero recientes progresos en las técnicas de ingeniería metabólica han propuesto posibilidades de enriquecimiento de alimentos mediante la biofortificación. Existen algunos avances en este sentido, con el uso de cepas recombinantes de bacterias con el incremento en la expresión de las enzimas 3 y 4 de la ruta (ascorbato decarboxilasa y pantoteato sintetasa) (Kupke et al. 2003; Rathinasabapathi \& Raman, 2005). Sin embargo, se requiere de mayor investigación en el tema, ya que esto puede llevar a la obtención de alimentos con amplias ventajas comparativas para la nutrición humana.

\section{Folatos en plantas: biosíntesis, distribución y enriquecimiento}

Los folatos son vitaminas que intervienen en la transferencia del carbono 1 (C1), en un juego de reacciones normalmente denominadas metabolismo C1. Químicamente, las moléculas de los folatos están compuestas por un grupo Pterin, ácido p- aminobenzoico (pABA) y una cadena de glutamato, con una variación entre 1 y 14 unidades (Rébeillé et al. 2006; Basset et al. 2005). Estos autores indican que el pool celular de folato está representado por una mezcla compleja de moléculas integradas, las cuales difieren en el estado de oxidación del anillo pterin (di o tetra hidroxifolato; $\mathrm{H}_{2} \mathrm{~F}$-Glun, $\mathrm{H}_{4} \mathrm{~F}$-Glun), en el estado de oxidación carbono 1 y en el número de moléculas de glutamato. Las unidades $\mathrm{C} 1$ son transportadas solamente por el $\mathrm{H}_{4} \mathrm{~F}$-Glun y pueden ser ligadas al nitrógeno 5 o al nitrógeno 10, en forma de puente.

Estas especies de folatos ofrecen unidades de carbono 1 para vías metabólicas que tienen una destacada impor- 
tancia para la viabilidad celular. Entre otros aspectos se resaltan: el 10 formil tetrahidrofolato es necesario para la síntesis de purinas y formal methionina tRNA; 5, 10 metilen dihidrofolato es necesario para la síntesis de thimidilato y pantotenato; 5-metiltetrahidrofolato, provee el grupo metil requerido para transformar homocysteina en mehtionina. El $80 \%$ de la methionina proviene de dicha vía. En plantas los folatos están involucrados en vías específicas, como la biosíntesis de clorofila y lignina (Cossins, 2000; Mouillon et al. 2002).

Para la salud humana, la deficiencia de folatos conduce a un sin número de enfermedades, como deficiencias en el sistema nervioso, central, enfermedades cardiovasculares, incrementos de homocisteina en el plasma, lo cual induce a riesgos por demencia, enfermedad de Alzheimer y esquizofrenia (Ravanel et al. 2001). La deficiente síntesis de methionina también da como resultado una deficiente cantidad de Adonet (S-adenosil- $L$-methionina), trascendental para la metilación del DNA. La hipometilación de las regiones promotoras de genes puede llegar a modificar la expresión genética, en especial para alterar la expresión de oncogénesis (Quinlivan et al. 2002). Entre otras consecuencias de la baja cantidad de folatos a nivel celular está la alta concentración de UMP respecto a TMP, debido a la limitación de la oferta de 5,10 metilen $\mathrm{H}_{4} \mathrm{~F}$-Glun. Esto da como resultado una alta incorporación de UTP dentro del DNA y la consecuente conformación de puntos mutantes, rompimiento sencillo o doble en las cadenas y, finalmente, rompimiento cromosómico. Este fenómeno está relacionado íntimamente con la aparición de cáncer colon-rectal, de hígado, pancreático, bronquial y cervical, así como leucemia (Rèbeillè et al. 2006).

\section{Biosíntesis de tetrafolatos en plantas}

La síntesis de folatos requiere de la participación de tres compartimentos celulares a saber: mitocondria, citosol y cloroplasto. Posiblemente, la vacuola sirva como órgano de almacenamiento de los productos (Mouillon et al. 2002). En el citosol, se requiere de una molécula de GTP para producir hidroximetildihidropterin, en tres pasos. La primera reacción es catalizada por GTP ciclohidrolasa I para formar dihidroneopterin trifosfato. Este producto es la base no solo para la producción de $\mathrm{H}_{4} \mathrm{~F}$-Glun sino para otros ptereines, una familia de coenzimas involucradas en una amplia variedad de reacciones que actúan como cromóforos. En el siguiente paso, la enzima nudix hidrolasa remueve el grupo pirofosfato y la dihidroneopterinaldolasa forma el hidrometildihidropterin (Hanson $\mathcal{E}$ Roje, 2001; Hanson E Gregory, 2002). Este compuesto entra a la mitocondria donde una pirofosfoquinasa une un grupo pirofosfato para producir $\mathrm{H}_{2}$ Pterin PPi. De otra parte, con base en una molécula de corismato en el cloroplasto, las enzimas aminodeoxicorismatosintasa y dihidroxicorismatoliasa forman ácido $p$-aminobenzoico, el cual sale del cloroplasto al citoplasma, luego entra a la mitocondria y allí se une con $\mathrm{H}_{2}$ Pterin, para formar $\mathrm{H}_{2}$ Pteroato, reacción catalizada por dihidropteroatosintasa. Posteriormente reincorporan, de forma sucesiva, los glutamatos para formar $\mathrm{H}_{4} \mathrm{~F}$-Glu $n$, mediante la dihidrofolatosintetasa. Este es el producto base de todos los folatos en plantas (Ravanel et al. 2001; Basset et al. 2005; Rèbeillè et al. 2006).

Los humanos necesitan consumir cuando menos $400 \mu \mathrm{g}$ de folatos en la dieta, pero en muy pocas ocasiones se logra alcanzar este valor (Hanson $\&$ Roje, 2001). En la taba 1 , se muestra el contenido de folatos en algunas especies agrícolas, con el fin de reflexionar sobre lo expuesto anteriormente. Es de anotar que las plantas dicotiledóneas y especialmente las leguminosas tienen altos contenidos de ácido fólico en sus semillas, hecho fundamental para seleccionar dietas apropiadas en humanos.

\section{Ácido ascórbico, biosíntesis y acumulación en plantas}

El l-ácido ascórbico (vitamina C) tiene el estatus de vitamina, debido a que algunos animales como primates, tienen poca habilidad para sintetizar este multifuncional cofactor enzimático. La mayor parte de la vitamina C es aportada por la dieta, siendo más alta la concentración en frutas y verduras. Se considera que en raras ocasiones la población humana sufre de deficiencia en vitamina $C$, a tal punto que no existen esfuerzos para enriquecer los alimentos con este compuesto, tal y como si ocurre con otros metabolitos de su misma naturaleza (Conklin, 2001)

Las plantas contienen una cantidad relativamente alta de ascorbato, donde tiene papel como regulador de diversas reacciones de oxidoreducción y cofactor enzimático. También tiene un papel interesante como fotoprotector, en respuestas hormonales, muerte celular programada, senescencia y ataque de patógenos (Jain 
Tabla 1. Contenido de folatos en algunas especies agrícolas. Valores expresados en nmol de ácido fólico por gramo de peso fresco (USDA, 2004).

\begin{tabular}{|c|c|}
\hline Producto & Contenido de folato $\left(\mathbf{n m o l ~}^{-1}\right)$ \\
\hline Arrozblanco & $0,13-0,18$ \\
\hline Trigo duro & $0,84-0,95$ \\
\hline Maízamarillo & 0,42 \\
\hline Tomate & $0,20-0,64$ \\
\hline Averjas verdes & 1,45 \\
\hline Hojas de espinaca & 4,31 \\
\hline Frijoles rosados & 10,28 \\
\hline Lentejas & 962 \\
\hline
\end{tabular}

E Nessler, 2000; Pallanca \& Smirnoff, 2000). Por consiguiente su biosíntesis está relacionada con fotosíntesis y respiración. En segundo lugar tiene importancia en la elaboración de alimentos, pues es un comodite de alto volumen y bajos costos utilizado como aditivo vitamínico en diferentes procesos.

\section{Biosíntesis}

En mamíferos, la biosíntesis se conoce desde los años 50. La UDP-glucosa derivada del glucógeno, se considera como la materia prima de la síntesis de novo del ácido ascórbico. En plantas, la biosíntesis es diferente, pues se supone que la base de la vía es la oxidación de L-Galactosa, la cual proviene de GDP-L manosa. La fosfomanosaisomerasa cataliza el primer paso de la reacción para formar D-manosa 1- fosfato, la cual con GDP forma GDP- d-manosa, con la consecuente liberación de pirofosfato. GDP-d-manosa forma GDP-l-galactosa mediante una epimerasa, luego se forma L-galactosa 1 -fosfato, mediante una fosforilasa. El producto de esta reacción, la l galactosa, se une con una molécula de L-galactonato para producir L-galactolactona, gracias a un producto proveniente de la pectina, denominado $D$-galacturonato. Proveniente del myo-inositol llega una molécula de gulonato, la cual se une con L-GalL y forma el L-ascorbato, mediante la acción de una deshidrogenada y una citocromooxidasa (Ishikawa et al. 2006; Loewus, 1999).

Se conoce que el contenido de ácido ascórbico en plantas está influenciado por la luz y varía durante las etapas de desarrollo. Se ha encontrado altos contenidos en hojas, en tallos, en frutos y, menores contenidos en semillas y en raíces. La falta de ácido ascórbico, en general, va a producir: disminución en la síntesis de ácido absícico, se afecta el crecimiento y la división celular, concentraciones altas de $\mathrm{H}_{2} \mathrm{O}_{2}$ Los altos contenidos de ascorbato, se relacionan con el incremento de resistencia al ataque de patógenos, especialmente virus (Smirnoff, 2000).

\section{La vitamina E: papel, biosíntesis y manipulación}

Los tococromanoles son moléculas amfifáticas, asociadas con lípidos de membrana, tienen una cabeza que mantiene la superficie de la membrana y un cuerpo completamente saturado, compuesto por 20 carbonos (Schneider, 2005). Tocoferoles y tocotrienoles hacen parte de este grupo de sustancias y difieren por el grado de saturación de los cuerpos hidrofóbicos. Los tocoferoles tienen un cuerpo carbonado completamente saturado proveniente del fitidilfosfato, mientras los tocotrienoles contienen un cuerpo derivado del GGDP y, por tanto, contienen tres enlaces dobles en los carbonos 3, 7 y 11 (Della Penna E Last, 2006). De acuerdo a lo expresado por Traber (2005), la vitamina $\mathrm{E}$ de forma activa, está compuesta por una mezcla de tococromanoles, donde la actividad principal se debe a los $\alpha$-tocoferoles Todos los tocoferoles sintetizados naturalmente son esteroisómeros, mientras que los sintetizados, de forma química, son mezclas racémicas, de ocho esteroisómeros, los cuales tienen un impacto negativo en la actividad de la vitamina $\mathrm{E}$. 
Los tejidos vegetales varían enormemente en el contenido de vitamina E. En general, en plantas fotosintéticamente activas y no estresadas, el contenido de tococromanoles está entre 10 y $50 \mu \mathrm{g} \cdot \mathrm{g}^{-1}$ de peso fresco, siendo predominantemente $\alpha$-tocoferol. Las semillas tienen una mayor cantidad de tococromanoles, en rangos de 300 a $2000 \mu \mathrm{g} \cdot \mathrm{g}^{-1}$ de aceite (Della Pena E Last, 2006). En la tabla 2, se presentan los contenidos de tocoferol en algunos productos agrícolamente aprovechables.

Tabla 2. Niveles de tocoferol y composición de algunos tejidos de plantas. Datos expresados en $\mu \mathrm{g} \cdot \mathrm{g}^{-1}$ de peso fresco 0 gramo de aceite (Hess, 1993).

\begin{tabular}{|c|c|c|}
\hline Planta y órgano & Total tocoferoles & A-Tocoferol \% \\
\hline Tubérculos de papa & 0,7 & 10 \\
\hline Arroz blanco & 17 & 30 \\
\hline Hojas de lechuga & 7 & 45 \\
\hline Hojas de espinaca & 30 & 63 \\
\hline Semilla de girasol & 700 & 96 \\
\hline Aceite de maíz & 1000 & 20 \\
\hline Gemen de trigo & 2700 & 47 \\
\hline
\end{tabular}

\section{Biosíntesis}

La síntesis de $\alpha$ - tocoferol y de otros tococromanoles, se lleva a cabo en el citosol a partir de aminoácidos aromáticos para sintetizar la cabeza del grupo y, a partir de un producto proveniente de los cloroplastos, el 2.C.metil- $D$ eritritol-4 fosfato (MEP), para la producción del tallo o cuerpo del compuesto. En el primer caso, a partir de la vía del ácido shikimico, se obtiene hidrixifenilpiruvato, el cual produce ácido gentísico. A partir de MEP, se obtiene GGDP, que con un grupo fitilmonofosfato proveniente de un fitol, produce fitidildifosfato (PDP). El PDP se une con HGA y forma 2-metil-6-fitil benzoquinol (MPBQ). Luego, el MPBQ se transforma en $\alpha$ - tocoferol (Cahoon et al. 2003; Horvatha et al. 2006).

Mirando hacia el futuro y con base en las cantidades variables de tocoferol en los alimentos, queda claro que se pueden alcanzar logros destacados en el mejoramiento de los productos agrícolas, mediante el incremento de las concentraciones de tocoferol. La ingeniería genética y la ingeniería metabólica son la base para poder incrementar los niveles del cofactor, sobre todo en plantas cuyos órganos de almacenamiento y aprovechamiento sean semillas o que contengan unas cantidades altas de aceites y grasas. No obstante, no se bebe desconocer que las técnicas de mejoramiento convencional han contribuido a mejorar la calidad de los alimentos y que siguen siendo herramientas muy importantes.

\section{Carotenoides en plantas y su importancia nutricional}

Con base en lo expuesto por Botella-Pavia E Rodríguez (2006), los carotenoides son isoprenoides lipofílicos sintetizados por organismos fotosintéticos, incluyendo plantas, algas y cianobacterias, pero también algunas bacterias y hongos. Se encuentran dos clases en la naturaleza: los carotenos que son hidrocarbonatos puros y las xantofilas, que contienen oxígeno. El $\beta$-caroteno, el cual ya se trató al principio del documento, tiene importancia por ser el precursor de la vitamina A. Dentro de las xantofilas, se encuentran la zeaxantina, muy especial para dar coloración al maíz, la violaxantina, la luteína y la neoxantina, entre otras. 
La mayoría de carotenoides se asocian con las clorofilas y son enmascarados por éstas. Cuando los tejidos fenecen y las clorofilas son oxidadas, se pede ver los caroteniodes y más aún se concentra su mecanismo de acción fisiológica (Hirschberg, 2001). Fisiológica y bioquímicamente tienen enorme interés, pues conforman la estructura fotosintética que permite la captación de luz, el transporte de electrones y la protección de fotosistemas y clorofilas para no ser fotodestruidos. La transformación oxidativa de los carotenoides da como resultado la producción de apocarotenoides, los cuales tienen una potente actividad biológica (Dawe et al. 2002). El ácido absícico y algunos otros fitoreguladores, aun no identificados, son los mejores ejemplos.

El principal papel de los carotenoides parece estar enmarcado en dar la coloración apropiada a las flores para atraer los polinizadores, para atracción de pájaros y así dispersar las semillas y, como complemento, dar coloración adecuada a flores y frutos para contribuir al incremento de su valor económico. Las propiedades de los carotenoides, como pigmentos agroindustriales, han sido explotadas desde hace bastante tiempo, ejemplo de ello es el uso de astaxantina y canthaxantina en la alimentación de salmones truchas y pargos para dar la coloración apropiada de su carne. Luteína y zeaxantina son usadas para dar una mejor coloración a huevos y carne de pollo, mientras que $\beta$-caroteno es usado en la coloración de margarinas (Cunningham \& Gantt, 1998).

Los carotenoides para animales y para el hombre son fundamentales y mucho más que pigmentos y precursores de vitamina A. De hecho, los carotenoides actúan como precursores de la producción de retinoides, como el retinol (vitamina A), retinal (principal pigmento visual) y ácido retinoico, el cual controla aspectos morfogenéticos. La deficiencia de $\beta$-caroteno en la alimentación humana es el principal problema de salud en el mundo, especialmente en países en desarrollo, causando problemas, como ceguera, xerophtalmia, muerte prematura de niños y enfermedades de las mujeres en gestación (Lucca et al. 2006).

\section{Biosíntesis}

Como todos los isoprenoides, los carotenoides son sintetizados de una unidad de isopreno, el isopentildifosfato (IPP) y su isómero, el dimetilalildifosfato (DMAPP). Tres moléculas de IPP forman GGDP, un precursor común de cierto grupo numeroso de isoprenoides, entre los cuales se encuentran giberelinas, clorofilas, tocoferoles, filoquinonas y plastoquinonas. La condensación de dos GGDP genera una cadena isoprenoide poliénica con dobles enlaces conjugados para estructurar la columna vertebral de los carotenoides vegetales y determinar sus propiedades biológicas (Hirschberg, 2001). Adelantos en la ingeniería genética han permitido encontrar los efectos anticancerígenos y las propiedades antioxidantes de estos compuestos. Es así que la demanda mundial por carotenoide, de uso industria, como zeaxantina, asthaxantina, canthaxantina y bixina ha aumentado y, por ello, el especial interés en algunos productos vegetales. En tomate, se ha incrementado las cantidades de licopeno y $\beta$-caroteno, en papa tanto las variedades de Solanum tuberosum como las de S. phureja han sido empleadas para incrementar los niveles de carotenoides con relativo éxito, pero tal vez los cultivos donde el mejoramiento genético ha aportado mayores expectativas son el arroz y la canola. El arroz, en el ya comentado caso del grano "Golden Rice", mientras que en canola, se han encontrado plantas transgénicas que han incrementado los niveles de $\beta$-caroteno hasta 300 veces respecto a los testigos (Botella-Pavia $E$ Rodríguez, 2006; Schaub et al. 2005).

\section{Importancia de los glucosinolatos}

Los glucosinolatos y los productos misceláneos derivados del rompimiento de su molécula fueron identificados inicialmente como aceites de coles y han despertado alta curiosidad. No solamente contribuyen al olor y al aroma característico de las especies crucíferas, sino que poseen profundas actividades biológicas, incluida su participación en mecanismos de defensa vegetal, homeóstasis auxínica, así como para la prevención del desarrollo de cáncer en humanos. Al encontrarse particularmente en Brassicaceas, grupo de plantas con varias especies de importancia económica, el estudio de estos metabolitos secundarios tiene especial interés para la agricultura (Padilla et al. 2007).

La estructura central de los glucosinolatos es la glucosa, la cual se deriva de un selecto grupo de aminoácidos proteicos y comprende un grupo $\beta$-tioglicosil, unido a un carbono $\alpha$, para formar una cetoxima sulfatada. La extensión y la modificación de la cadena de los glucosinolatos es la responsable de la diversidad química de más de 120 estructuras reportadas. Esos compuestos hidrofílicos e inestables son almacenados en las vacuolas de los diferentes tejidos vegetales. Una enzima conocida como mirosinasa, es la responsable de romper los 
glucosinolatos, mediante hidrólisis del grupo glicosídico (Chen E Andreason, 2001).

Las mirosinasas son $\alpha$-htioglucosinasas localizadas en el idioblasto (células mirosin), agrupadas en diversos tejidos de las plantas productoras de glucosinolatos. Daños por agentes bióticos y/o abióticos activan el sistema binario glucosinolato mirosina, algunas veces llamada la bomba de los aceites de coles, conduciendo a la formación rápida de un producto inestable llamado thiohidroxinato-O-sulfato. Posteriormente, la eliminación monoenzimática del grupo sulfato y el arreglo espontáneo del corazón de la estructura, producen una variedad de productos bioactivos incluyendo isohtiacinolatos (de alta presencia en brócoli y coliflor), thiocinatos, nitrilos, oxazolidionas y epitilalcanos. Las condiciones químicas, como $\mathrm{pH}$, disponibilidad de iones hierro y la presencia de mirosidinas, determinan la composición de la mezcla final de los productos. Algunos productos de la degradación tienen una potente actividad biocida y contribuyen ampliamente al mecanismo de defensa de las plantas; otros juegan papel como aleloquímicos en la interacción insecto-planta (Tripathi \& Mishra, 2007).

Como componentes de comidas y de alimentos, los productos de la hidrólisis de los glucosinolatos tienen importancia toxicológica y farmacológica. Algunos derivados de glucosinolatos tienen propiedades antinutricionales. En la actualidad, se conoce que los isotiocianatos derivados de la methionina tienen actividad anticancerígeno (Jones et al. 2006).

\section{Biosíntesis}

Según Fischer E Bacher (2006) y Grubb \& Abel (2006), la biosíntesis de glucosinolatos se divide en tres partes: elongación de la cadena de aminoácidos, desarrollo de la estructura central y modificaciones secundarias a la cadena. La primera, se inicia con una transaminación, seguida de una condensación del ceto-ácido y la coenzima A, para formar 2-alkil malato. La metiltioalkilmalatosnitetasa cataliza esta reacción. La conformación de los dos pasos siguientes, isomerización y carboxilación, es análogo a lo encontrado en el ciclo de los ácido tricarboxílicos. El resultado es un homo cetoácido, el cual sufre una trans aminación para formar un homoaminoácido. Las enzimas para estas reacciones no se han identificado aún. La formación de la glucona o estructura central, toma el homo amino ácido y lo transforma en thiohidroxinato mediante dos oxidaciones seguidas y una conjugación de con cysteina, mediante la ación de C-S lyasa. Finalmente, se forma glucosinolato a partir de thio hidroximato, UDP-glucosa y una sulfatación. En las modificaciones a la cadena, se presentan sucesivas oxidaciones, mediante la acción de alkenilfosfatooxidasa, lo cual generará grupos alkenil glucósido e hidroxil glucósido.

\section{Presencia en los alimentos}

En el brócoli y coliflor la presencia de glucosinolatos reviste especial importancia, gracias a los descubrimientos que han permitido identificar a dichas plantas como atenuadores de los riesgos por cáncer de estómago, de páncreas, de hígado, de colón y de próstata (Padilla et al. 2007; De Pascale et al. 2007)). De acuerdo a las investigaciones de Jones et al. (2006), el 4-metilsulfinilbutil es el derivado de mayor presencia. Este compuesto de los isothiosinatos tiene importantes propiedades como anticancerígeno. En la tabla 3, se observa la composición del brócoli en cuanto a derivados de glucosinolatos. Es claro que la cocción prolongada puede afectar el contenido de estos productos, de ahí que se recomiende hervirlos durante no más de cuatro minutos para mantener sus propiedades (De Pascale et al. 2007).

\section{El vino y sus componentes}

Los componentes fenólicos constituyen uno de los más importantes parámetros de calidad de los vinos, pues ellos contribuyen a las características organolépticas, particularmente color, sabor y astringencia. Estos componentes, se dividen en dos grupos: no flavonoides donde se encuentran el ácido hidroxibenzoico, el ácido hidroxicinámico, los estilbenos y los alcoholes fenólicos; el otro grupo denominado flavonoides donde se encuentran las antocianinas, los flavonoles, los flavanos y los dihidroflavonoles (Monagas et al. 2006). Los estilbenos, así como sus polifenoles oligoméricos constituyen un grupo de sustancias naturales, las cuales son producidas como metabolitos en plantas sometidas a estrés, por lo cual, se considera que hacen parte del arsenal de defensa de los vegetales, siendo clasificados como fitoalexinas (Dourtoglou et al. 1999).

Trans-resveratrol (3, 5, 4-trihidroestilbeno) es una fitoalexina de la vid, que actúa como un antioxidante de lipoproteínas de baja densidad en humanos. Se encuentra en la cáscara del fruto de la uva. Su isómero el cis-resveratrol es poco común en condiciones naturales y se presencia es derivada de procesos químicos. Se 
Tabla 3. Composición de derivados de glucosinolatos en la inflorescencia del brócoli (Brassica oleracea var italica) (Jones et al. 2006).

\begin{tabular}{|c|c|c|c|}
\hline Nombre común & Nombre químico & Clase & Abundancia \% \\
\hline Glucurafanin & 4-metilsulfinilbutil. & Alifático & 55,5 \\
\hline Glucobrassicin & 3-indolmetil & Indolil & 8,6 \\
\hline Gluconapin & 3-butenil & Alkenil & 7,8 \\
\hline Prpgroitin & (2R) 2-hydroxi-3-butenil & Alkenil & 7,8 \\
\hline Napoleiferin & 2-hidroxi-4-pentinil & Indolil & 5,5 \\
\hline 4-metoxiglucobrassicin & 4-metoxi-3indolmetil & Indolil & 3,1 \\
\hline Gluconasturtin & 2-feniletil & Aromático & 3,1 \\
\hline Glucobrassicanapin & 4-pentinil & Indolil & 2,3 \\
\hline Glucoalisin & 5-Metilsulfinilpentil & Alifático & 1,6 \\
\hline Neoglucobrassin & N-metoxy-3-indolmetil & Indolil & 1,6 \\
\hline
\end{tabular}

identificó inicialmente en tejidos afectados por infecciones de los hongos Botrytis cinerea y Plasmophora vitícola. La presencia de trans-resveratrol en vinos es especialmente importante por sus características antinflamatorias y anticoagulantes, las cuales pueden proteger contra arteriosclerosis y enfermedades coronarias. Las concentraciones de la fitoalexina son mayores en vinos rojos procesados a partir de la maceración de la cáscara de la uva, mientras que se han encontrado bajas concentraciones en vinos blancos obtenidos de la fermentación de pulpas. Las variedades de vid, las condiciones climáticas, las prácticas culturales y el añejamiento en toneles de roble también afectan los niveles del estilbeno en el producto final.

Si se tiene en cuenta que en el mundo una de cuatro personas puede padecer de algún tipo de cáncer es importante pensar en el uso del resveratrol como un agente quimiopreventivo de dicha enfermedad, pues bloquea la agregación de plaquetas, lo cual permite también disminuir los riesgos por ataques cardiacos y leucemia (Gerogianaki et al. 2006). En la tabla 4, se puede observar

Tabla 4. Contenido de trans-resveratrol en clases de vinos. Datos tomados de Geragiinaki et al. (2006).

\begin{tabular}{|c|c|c|}
\hline VARIEDAD & TIPO & Trans-Resveratrol en mg f-1 $^{-1}$ \\
\hline Savatiano & Blanco & 0,571 \\
\hline Muskat De Alejandria & Blanco & 0,015 \\
\hline Vilana & Blanco & 0,211 \\
\hline Cabemet & Rojo & 0,741 \\
\hline Agiorgitico & Rojo & 1,213 \\
\hline Mantilaria & Rojo & 1,991 \\
\hline
\end{tabular}


los contenidos de trans-resveratrol en algunas variedades de vid y las características de los vinos.

\section{Café y chocolate, dos bebidas de alto impacto}

El café y el chocolate son dos de las bebidas de origen natural más importantes en el mundo. Esta importancia es mayor si se tiene en cuenta la participación de los productos base de las bebidas en la economía colombiana. Los contenidos de cafeína y theobromina en las bebidas los han puesto en la mira de muchos contradictores, pero también de varios defensores de sus propiedades.

El cacao y su derivado el chocolate, desde tiempos precolombinos han sido considerados alimentos de gran valor energético y nutricional en la dieta de los habitantes de las culturas meso Americanas. Recientemente, se ha descubierto que pueden ser otras fuentes de transresveratrol, diferentes a los vinos tintos (Counet et al. 2006). Este aspecto es de vital importancia, debido a que el consumo de chocolate puede mejorar la condición saludable de los humanos, especialmente para evitar enfermedades de tipo coronario.

La cafeína (1,3,7 trimetilxantina) es uno de los pocos productos vegetales, con los cuales la mayoría de población está familiarizada, debido a la presencia de ella en algunas bebidas, como té, café y algunas gaseosas. Un concepto creciente, basado en los efectos adversos de la cafeína en la salud humana, ha disparado el consumo de productos llamados descafeinados (Parras et al. 2007; McCusker et al. 2006). El consumo de cafeína, produce entre otras cosas, palpitaciones excesivas, desórdenes gastrointestinales, ansiedad, temor, incremento en la presión sanguínea e insomnio (Ashinara \& Crozier, 2001). Descubierta inicialmente en el té (Camellia sinensis) y café (Coffea arabica) hace parte del grupo de las mehtilxanthinas, junto con la theobromina del cacao, paraxanthina y ácido metil úrico, conocidos como los alcaloides de purina (Zheng et al. 2004). Los alcaloides de purina están también presentes en hojas de mate (Ilex paraguarienses), guaraná (Paulliana cupana), cola (Cola nitida), en flores de cítricos y en metabolitos de hongos, como el caso de los esclerocios de Claviceps purpurea.

\section{Biosíntesis de los alcaloides de purina}

La xanthosina es el compuesto purínico inicial para la síntesis de cafeína, actuando como un sustrato del grupo methil donado por S-adenosil-Methionina (SAM). Producto de esto, se obtiene la 7-methilxanthosina, luego 7-methilxanthina, thebromina y finalmente cafeína, aunque algunas rutas alternas de menos ocurrencia pueden darse. Una vez alcanzada la síntesis de cafeína, la xanthosina se convierte en xanthina para ser degradada a $\mathrm{CO}_{2}$ y $\mathrm{NH}_{3}$, como vía catabólica (Ashinara $\&$ Crozier, 2001; Koyama et al. 2003).

\section{CONCLUSIONES}

Para realizar aportes al problema de la seguridad alimentaria mundia,l en especial en los países en vía de desarrollo, es importante pensar en estrategias económicas y a largo plazo. Una de las posibilidades radica en la biofortificación de alimentos que hacen parte de la cultura de los pueblos y el mejoramiento de la calidad de los mismos. La transgénesis, la biotecnología y la fotoquímica, se deben unir e integrar para producir alimentos cada vez más completos y menos nocivos para la salud humana.

En el mundo existen muchos trabajos sobre mejoramiento de la calidad de los alimentos base de la población humana, pero lamentablemente la información no llega a los puntos neurálgicos de la tecnología, a los gobiernos y las entidades responsables de las políticas alimentarias.

Se debe mirar con bastante detalle las costumbres de los pueblos y con base en ellas aplicar los adelantos científicos que, en términos de metabolismo secundario $\mathrm{y}$ alimentos, se han expuesto en el presente trabajo.

\section{BIBLIOGRAFÍA}

AL-BABILI, S.; BEYER, P. 2005. Golden Rice-five years on the road-five years to go? Trends Plant Sci. 10(12):565-572.

ASHINARA, H.; CROZIER, A. 2001. Caffeine: a well known but little mentioned compound in plant science. Trends Plant Sci. 6(9):407-414.

BACHER, A.; EBERHARDT, S.; FISHER, M.; KIS, K.; RICHTER, G. 2000. Biosynthesis of vitamin $B_{2}$ (rivoflavin). Ann. Rev. Nutr. 20:153-157. 
BASSET, G.J.C.; QUINLIVA, E.P.; GREGORY, J.F. III.; HANSON, A.D. 2005. Folate synthesis and metabolism in plants and prospects for biofortification. Crop Sci. 45:449-453.

BOUIS, H.E. 2003. Micronutrient fortification in plants through plant breeding. Can it improve nutrition in man at low cost? Proc. Nutr. Soc. 62:403-411.

BOTELLA-PAVIA, P.; RODRÍGUEZ, C.M. 2006. Carotenoid biotechnology in plants for nutritionally improved foods. Physiol. Plant. 126:269-381.

CAHOON, E.B.; HALL, S.E.; RIPP, K.G.; GANZKE, T.S.; HITZ, W.D. 2003. Metabolic redesign of vitamin E biosynthesis in plants for tocotrienol production and increased antioxidant content. Nat. Biotech. 21:1082-1087.

CHAKAUYA, E.; COXON, K.; WHITNEY, H.M.; ASHURRST, J.L.; ABEL, C.; SMITH, A.G. 2006. Pantothenate biosynthesis in higher plants: advances and challenges. Physiol. Plant. 126:319-329.

CHEN, S; ANDREASSON, E. 2001. Update on glucosinolate metabolism and transport. Plant Physiol. Biochem. 39:743-758

CONKLIN, P.L. 2001. Recent advances in the role and biosynthesis of ascorbic acid in plants. Plant Cell Environ. 24:383-394.

COSSINS, E.A.; 2000. The fascinating world of folates and one carbon metabolism. Can. J. Bot. 78:691708.

COUNET, C.; CALLEMIEN, D.; COLLIN, S. 2006. Chocolate and cocoa: New sources of trans-resveratrol and trans-piceid. Food Chem. 98(4):649-657.

CUNNINGHAM, F.X.; GANTT, E. 1998. Genes and enzymes of carotenoid biosynthesis in plants. Ann. Rev. Plant Physiol. Plant Mol. Biol. 49: 557-583.

DATTA, K.; BAISAKH, N.; OLIVA, N.; TORRIZO, L.; ABRIGO, L. 2003. Bioengineered "golden" indica rice cultivars with $\beta$-carotene metabolism in the endosperm with higromicin and mannose selection systems. Plant Biotech. J. 1:81-90.
DAWE, D.; ROBERTSON, R.; UNNEVERTH, L. 2002. Golden rice: what role could it play in alleviation of vitamin A deficiency? Food Policy 27:541-560.

DELLA PENNA, D.; LAST, R. 2006. Progress in the dissection and manipulation of plant vitamin $\mathrm{E}$ biosynthesis. Physiol. Plant. 126:356-368.

DE PASCALE, S.; MAGGIOA, A.; PERNICE, R.; FOGLIANO, V; BARBIERI, G. 2007. Sulphur fertilization may improve the nutritional value of Brassica rapa L. subsp. sylvestris. Eur J. Agron. 26:418-424.

DOURTOGLOU, V.G.; MAKRIS, D.P.; BOIS-DONNAS, F.; ZENOAS, C. 1999. Trans-Resveratrol concentration in wines produced in Greece. J. Food Comp. Anal. 12:227-233.

DUHAN, A.; KHETARPAUL, N.; BISHNOI, S. 2002. Content of phytic acid and $\mathrm{HCl}$-extractability of calcium phosphorus and iron as affected by various domestic processing and cooking methods. Food Chem. 78:9-14.

FISCHER, M.; BACHER, A. 2005. Biosynthesis of flavocoenzymes. Nat. Prod. Report. 22:324-350.

FISCHER, M.; BACHER, A. 2006. Biosynthesis of vitamin $\mathrm{B}_{2}$ in plants. Physiol. Plant. 126:304-318.

GEROGIANNAKI, M.; ATHANASOPOLUS, P.; KYRIAKIDIS, N.; GEROGIANNAKI, E.; SPANOS, $M$. 2006.Ttrans-resueratrol in wine from the major greek red and white grape varieties. Food Cont. 17:700-706.

GHANDILYAN, A.; VREUGDENHIL, D.; AARTS, M.G. 2006. Progress in the genetic understanding of plant iron and zinc nutrition. Physiol. Plant. 126:407-417.

GRUBB, C.D.; ABEL, S. 2006. Glucosinolate metabolism and its control. Trends Plant Sci. 11(2):89-91.

HANSON, A.D.; ROJE, S. 2001. One-carbon metabolism in higher plants. Ann. Rev. Plant Physiol. Plant Mol Biol 52:119-137. 
HANSON, A.D.; GREGORY, J.F. 2002. Synthesis and turnover of folate in plants. Curr. Opin. Plant. Biol. 5:244-249.

HESS, J.L. 1993. Vitamin E, tocopherol. En: Alscher, R.; Hess, J. edits. Antioxidants in higher plants. CRC Press Boca Ratón, p.111-134.

HIRSCHBERG, J. 2001. Carotenoid biosynthesis in flowering plants. Curr. Opin. Plant Biol. 4:210-218.

HORVATHA, G.; WESSJOHANNB, L.; BIGIRIMANAC, J.; MONICAC, H.; JANSEND, M.; GUISEZA, Y.; CAUBERGSA, R.; HOREMANSA, N. 2006. Accumulation of tocopherols and tocotrienols during seed development of grape (Vitis vinifera L. cv. Albert Lavallée). Plant Phys. Biochem. 44:724-731.

ISHIKAWA, T.; DOWDLE, J.; SMIRNOFF, N. 2006. Progress in manipulating ascorbic acid biosynthesis and accumulation in plants. Physiol. Plant. 126:343-355.

JAIN, A.K.; NESSLER, C.L. 2000. Metabolic engineering of an alternative pathway for ascorbic acid in plants and animals. Mol. Breed. 6:73-78.

JOHNS, T.; EYZAGUIRRE, P.B. 2007. Biofortification, biodiversity and diet: A search for complementary applications against poverty and Malnutrition. Food Pol. 32:1-4.

JONES, R.B.; FARAGHER, J.D.; WINKLER, S 2006. A review of the influence of postharvest treatments on quality and glucosinolate content in broccoli (Brassica oleracea var. italica) heads. Postharvest. Biol. Tech. 41:1-8.

KING, J.C. 2002. Evaluating the impact of plant biofortification on human nutrition. J. Nutr. 132:511-513.

KOYAMA, Y.; TOMODA, Y.; KATO, M.; ASHIHARA, H. 2003. Metabolism of purine bases, nucleosides and alkaloids in theobromine-forming Theobroma cacao leaves. Plant Physiol. Biochem. 41:997-984.

KUPKE, T.; HERNÁNDEZ-ACOSTA, P.; CULIÁÑEZMACIA, F. A. 2003. A-phosphopanteteina and coenzime A biosynthesis in plants. J. Biol. Chem. 278:38229-38237.
LOEWUS, F.A. 1999. Biosynthesis and metabolism of ascorbic acid in plants and of analogs of ascorbic acid in fungi. Phytochem. 52:193-210.

LUCCA, P.; POLETTI, S.; CAUTTER, C. 2006. Genetic engineering approaches to enrich rice with iron and vitamin A. Physiol. Plant. 126:291-303.

LÓPEZ, M.A.; MARTOS, F.C. 2004. Iron availability: An updated review. Internal. J. Food Sci. Nutr. 55:597-606.

McCUSKER, J.; RACHEL R.; FUEHRLEIN, B.; GOLDBERGER, B.A.; GOLD, M.S.; CONE, E. 2006. Caffeine content of decaffeinated coffee. J. Anal. Tox. 30(8):611- 613.

MENG, F.; WEI, Y.; YANG, X. 2005. Iron content and biolavailability in rice. J. Trace Elem. Med. Biol. 18:333-338.

MONAGAS, M.; GÓMEZ, C.C.; BOGOÑA, B. 2006. Evolution of the phenolic content of red wines from Vitis vinifera L. during ageing in bottle. Food Chem. 95:405-412.

MOUILLON, J.M.; RAVANEL, S.; DOUCE, E.; REBEILLE, F. 2002. Folate synthesis in higher-plant mitochondria: coupling between the dihydropterin pyrophosphokinase and the dihydropteroate synthase activities. Biochem. J. 363:313-319.

OLTMANS, S.E.; FEHR, W.R.; WELKE, G.A.; RABOY, V.; PETERSON, K.L. 2005. Agronomic and seeds traits of soybean lines with low-phytate phosphorus. Crop Sci. 45:593-598.

PADILLA, G.; CARTEA, M.E.; VELASCO, P.; DE HARO, A.; ORDÁS, A. 2007. Variation of glucosinolates in vegetable crops of Brassica rapa. Phytochem, 68(4):536-545.

PALLANCA, J.E.; SMIRNOFF, N. 2000. The control of ascorbic acid synthesis and turnover in pea seedlings. J. Exp. Bot. 51:669-674.

PARRAS, P.; MARTÍNEZ-TOMÉ, M.; JIMÉNEZ, A.M.; MURCIA, M.A. 2007, Antioxidant capacity of coffees of several origins brewed following three different procedures. Food Chem. 102(3):582-592. 
QUINLIVAN, E.P.; MC PARLIN, J.; MCNULTY, H.; WARD, M.; STRAIN, J.J.; WEIR, D.G. 2002. Importance of both folic acid and vitamin $B_{12}$ in reduction of risk of vascular disease. Lancet. 359:227-228.

RAVANEL, S.; CHEREST, H.; JABRIN, S.; GRUNWALD, D. 2001. Tetrahydrofolate biosynthesis in plants: molecular and functional caracterization of dihydro folate synthetase and three isiforms glutamate synthetase in Arabidopsis thaliana. Proc. Nat. Acad. Sci. 98:15360-15365.

RATHINASABAPATHI, B.; RAMA, S.B. 2005. Exogenous supply of pantonyl lactone to excised leaves increases their pantothenate levels. Ann. Bot. 95:1033-1037.

RÈBEILLÈ, F.; RAVANEL, S.; JABRIN, S.; DOUCE, R.; STOROZHENKO, S.; VAN DER STRATEN, D. 2006. Folates in plants: biosynthesis, distribution and enhacement. Physiol. Plant. 126:330-342.

SCHAUB, P.; AL-BABILLI, S.; DRAKE, R.; BEYER, P. 2005. Why is golden rice yellow instead of red? Plant Physiol. 138:441-450.

SCHNEIDER, C. 2005. Chemistry and biology of vitamin E. Mol. Nutr. Food Res. 49:7-30.

SCHRAMEK, N.; BRACHER, A.; BACHER, A. 2001. Biosynthesis of riboflavin. Single turnover kinetic analysis of GTP cyclohydrolase II. J. Bio. Chem. 276:44157-44162.

SMIRNOFF, N. 2000. Ascorbic acid: metabolism and functions of a multi facetted molecule. Current Op. Plant Biol. 3:229-235.
TRABER, M.G. 2005. Vitamin E regulation. Current Op. Gastroenterology 21:223-227.

TRIPATHI, M.K.; MISHRA, A.S. 2007. Glucosinolates in animal nutrition: A review. Anim. Feed Sci. Tech. 132(1,2):1-27.

USDA. 2004. USDA National Nutrient Database for Standard Reference. Disponible desde Internet en: http://www.nal.usda.gov/fnic/foodcomp/search (con acceso 12/05/06).

WEBB, M.B.; SMITH, A.G.; ABELL, C. 2004. Biosynthesis of pantothenate. Nat. Prod. Rep. 21:695-721.

WELCH, R.M.; GRAHAM, R.D. 2004. Breeding for micronutrients in staple food crops from a human nutrition perspective. J. Exp. Bot. 55:353-364.

WHITE, P. J.; BROADLEY, M.R. 2005. Biofortifying crops with essential mineral elements. Trends Plant Sci.. 10(12): 586-593.

XIAO, L.Y.; WU, P.; LING, H.Q.; XU, G.H.; XU. F.; ZHANG, Q. 2006. Plant nutriomics in China: An Overview. Ann. Bot. 98(3):473-48.

ZHENG, X-Q.; KOYAMA, Y.; NAGAI, C.; ASHIHARA, H. 2004. Biosíntesis, accumulation and degradation of theobromine in development Theobroma cacao fruits. J. Plant Physiol. 161:363-369.

Recibido:Noviembre 27 de 2006

Aceptado: Mayo 9 de 2007 